\title{
تأثيراضافة الفحم الحيوي (biochar) الى التبن المعامل او غير المعامل باليوريا على الصفات الاتتاجية وبعض صفات الأبيحة في الحملان العواسي

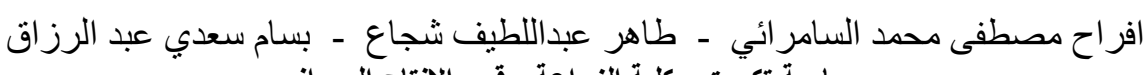 جامعة تكريت ـ كلية الزراعة ـ قسم الإتتاج الحيو اني
}

استخدم في هذه التجربة 16 حملاً عواسياً نراوحت أعمارها ما بين7_8 أثنهر وبمعدل وزن 27.55ـ 0.78 كجم، وزعت الحملان إلى أربعة مجاميع تغذوية حسب الوزن وفي كل مجموعة 4 حملان ثم وزعت المعاملات عشوائياً على المجاميع. الحملان غذيت على عليقة مركزة بنسبة3.5 \% من وزن الجسم , وكانت المعاملات كما يلى. المعاملة الأولى = عليقة مركزة + 0.5\% فحم حيوي+ تبن معامل باليوربا. المعاملة الثانية = عليقة مركزة + 0.5\% فحم حيوي + تبن غير معامل باليوريا.

المعاملة الثالثة= عليقة مركزة + zero\% فحم حيوي + تنبن معامل باليوربا. المعاملة الرابعة= عليقة مركزة + zero فحم حيوي + نبن غبر معامل بالبوريا. حلت النتائج وفق التصميم العشوائي في تجربه عامليه

أشارت نتائج التحليل الإحصائي إلى عدم وجود فروق معنوية لتأثير الفحم الحيوي او للتبن المعامل باليوريا او للتداخل بينهما في (معدل الوزن النهائي ومعدل الزيادة الوزنية الكلية ومعدل الزيادة الوزنية اليومية ومعدل استهلاك العلف وكفاءة التحويل الغذائي). كذلك عدم وجود فروق معنوية في بعض صفات الذبيحة للحملان العواسٍِ(نسبة التصافي ومساحة العضلة العينية )بالرجم من وجود اختلافات معنويه في وزن الذبيحة الحار والبارد ونسبة الدهن تحت الجلد للمعاملة الرابعة مقارنة بالمعاملة الاولى. الكلمات المفتاحية: الفحم الحيوي ، التبن المعامل باليوريا، الحملان العو اسية، صفات الذبيحة.

المقدمة

ان الهدف الأساسي من عملية تربية الأغنام هو انتاج اللحوم وبأقل كلفة لذا يلجا المربون الى تقديم الغذاء المناسب لحيواناتهم كما ونوعا لسد احتباجاتها الغذائية (طه واخرون، 1980) ، حيث تظهر اهمية استخدام التبن المتوفر بكميات كبيرة في العراق كعلف خشن في علائق الحيوانات المجترة بالرجم من انخفاض قيمته الغذائية لاحتوائه على نسبة عالية من اللكنين وهذا يؤدي الى انخفاض معامل هضمه ( حسن، 2005) ـ ولزيادة كمية المتتاول من التبن يتطلب تحسين القيمة الغذائية له ورفع معامل الهضم باستخدام وسائل عديدة ومختلفة منها المعاملات الكيميائية (المعاملة باليوريا والهيدروكسيدات) (توفيق، 2004; حسن و محد ،2008). ان اضافة الفحم الحيوي مع التبن المعامل باليوريا لغرض تحديد تحرير النيتزوجين وإمكانية استغلاله من قبل الاحياء المجهرية وامتزاز الفائض من قبل الفحم الحيوي وكذلك المحافظة على التلوث الحاصل من جراء طرح اليوريا من قبل الحيوانات وطرح الميثان الذي قد يؤدي الى التأثير على الغلاف الجوي الذي يؤدي الي ارتفاع اعداد الاحباء المجهرية بتكوين

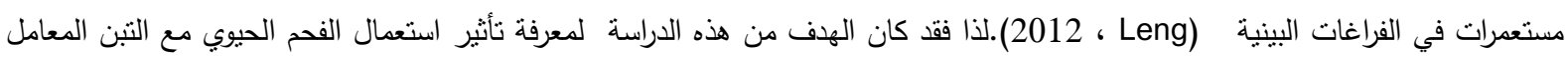
باليوريا وغير المعامل في الأداء الإنتاجي للحملان العواسية وبعض وصفات الذبيحة.

أجريت هذه الدراسة في حقل الاغنام التابعة لقسم الإنتاج الحيواني - كلية الزراعة / جامعة تكريت للفترة من (11/10 /2018) حتى

مواد وطرائق العمل (2019/2/23) بعد اكمال الفترة التمهيدية البالغة (15) يوما. واستخدم فيها 16 حملا عواسيا تم شرائها من الاسواق المحلية تراوحت اعمارها من (7-7) أثهر ومعدل اوزانها (27.55 (0.785) كجم وغذيت بصورة جماعية. نم وضع الحملان في اقفاص ابعادها (2×4 م). تم توزيع الحيوانات الى أربع مجاميع وحسب الوزن حيث كانت معدلات اوزان كل مجموعة مثقاربة مع بعضها وتضمنت كل مجموعة أربعة مكررات وبعدها نم نوزيع المعاملات عشوائياً على المجاميع الأربعة وقد نم تحليل النتائج وفق التصميم العشوائي لتجربة عامليه (2×2 ) وخضعت الحيوانات لفترة تمهيدية لمدة (15) يوم قبل البدء بالتجربة ـ قدم العلف لمرنين في البوم بنسبة 3.5\% من وزن الجسم وتم تعديل الكمية اليومية من العلف كل أسبوع بحسب 
الوزن الجديد للحيوانات، استمرت عملية الوزن كل أسبوع وبشكل دوري حيث تصوم الحيوانات ويرفع العلف مساءا وتوزن قبل تقديم العلف صباحا

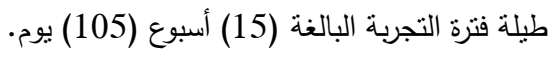

جدول 1. مكونات عليقة التجربة (جم/كجم).

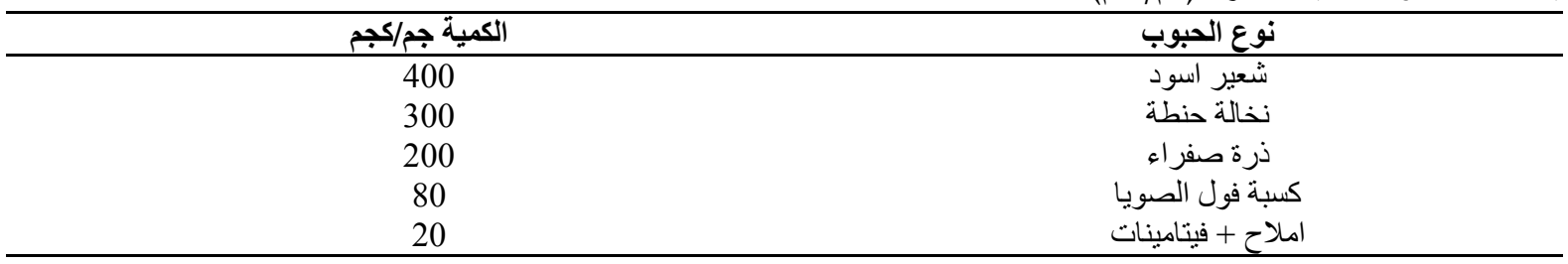

التحليل الكيميائي :تم تحليل عينات العلف وقدرت المادة الجافة والمادة العضوية والبروتين الخام والدهن الخام والألياف الخام وفق الطرق الكيمياوية

$$
\text { المستعملة في A.O.A.C (1990). }
$$

جدول 2. التركيب الكيميائي للعليقة المركزة وتبن الحنطة المعامل باليوريا وغير المعامل باليوريا (جم/كجم) مادة جافة:-

\begin{tabular}{|c|c|c|c|}
\hline \multirow[t]{2}{*}{ تبن حنطة معامل باليوريا 3\% } & \multirow[t]{2}{*}{ تبن حنطة } & \multirow[t]{2}{*}{ عليقة مركزة } & نوع العليقة \\
\hline & & & التركيب الكيميائي* \\
\hline 938.5 & 942 & 951 & المادة العضوية \\
\hline 124 & 25.4 & 142 & البروتين الخام \\
\hline 485 & 420 & 79 & الالياف الخام \\
\hline 33.5 & 21.8 & 41 & مستخلص الايثر \\
\hline 61.5 & 58 & 49 & 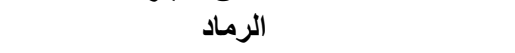 \\
\hline 296 & 475 & 689 & المستخلص الخالي من النتروجين \\
\hline 9.095 & 9.729 & 13.016 & الطاقة المتأيضةً MJ/kg * \\
\hline
\end{tabular}

$(1984, \mathrm{MAFF}) \mathrm{ME}=(\mathrm{NFE} * 0.014+\mathrm{CP} * 0.012+\mathrm{CF} * 0.005+\mathrm{EE} * 0.031) * *$

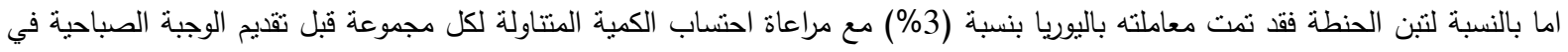

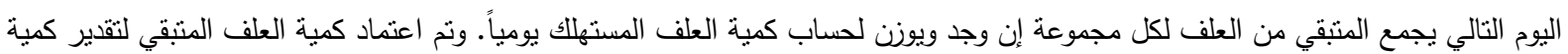

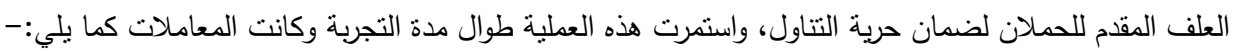

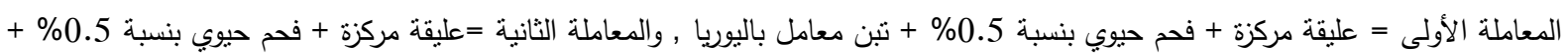

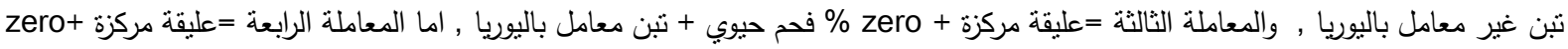
\% فحم حيوي + تبن غير معامل باليوريا.

\section{ذبح الحيوانات:}

عند انتهاء مدة التجربة رفع العلف عن الحملان لمدة 12 ساعة قبل عملية الذبح مع إبقاء ماء الثرب متوفر امام الحملان بصورة مستمرة وبعدها وزنت لغرض تثبيت الوزن عند الذبح (الوزن النهائي) وذبحت 8 حملان بواقع حملان من كل معاملة وسجلت أوزان الذبائح الحارة مشتملة على الكليتين ودهنهما (Field وآخرون ، 1963). حسبت نسبة التصافي على أساس:

$$
\text { نسبة التصافي = (الوزن الحار للإبيحة / وزن الجسم الفارغ للحيوان ) × } 100
$$$$
\text { نسبة التصافي = ( الوزن البارد للإييحة / وزن الجسم الفارغ للحيوان ) × } 100
$$

لضمان دقة اكبر في احتساب نسبة تصافي عن طريق إزالة او تقليل الخطأ الناتج عن محتويات الجهاز الهضمي. لهاردي. 
قياس مساحة العضلة العينية وسمك طبقة الدهن:

رسمت العضلة العينية Longissimusdorsi باستعمال ورق شمعي شفاف trace paper وقلم كر افيت ( Riley و آخرون ، 1966 ) وقيست مساحتها بو اسطة جهاز Planimeter الخاص بقياس المساحات غير المنتظمة ـو قيس أيضا سمك طبقة الدهن فوق الضلع الثاني عشر بواسطة جهاز digital Vernier إذ أخذت قر اءتين للجهة اليسرى . اسـتخدم البرنامج الإحصـائي المعامل باليوريا وتداخلهما في الصـــفات المدروســــة وفق تجربة عامليه طبقت بتصــــميم عثـــوائي كامل(2×2)، وقورنت الفروق المعنوية بين المتوسطات باختبار Duncan (1955) متعدد الحدود، وفق الأنموذج الرياضي الأتي:

$Y i j k=\mu+F i+T i+F T(i j)+e i j k$

أذ أن:

.k بijk بر: المتوسط العام للصفة المدروسة.

Fi تأثير العامل:Ti

Tj Ti

FT(ij)

eijk الخطأ العشو ائي الذي يتوزع توزيعا طبيعيا بمتوسط يساوي صفر وتباين قدره Ho

النتائج والمناقشة

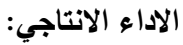

اشارت نتائج التحليل الاحصائي تأثير الفحم الحيوي في الجدول رقم (3) الى عدم وجود فروقات معنويه بين مجموعتي الحملان المقدم لها

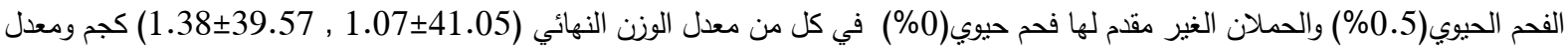

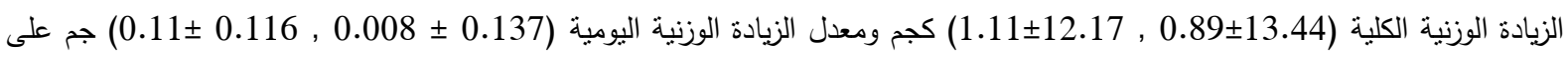
التوالي، وجاءت هذه النتائج مختلفة مع Preston و silivong (2015) , محمد امين و شجاع (2019) , وHang واخرون (2019) حيث وجدوا فروقات معنويه في معدل الزيادة الوزنية عند اضافه الفحم الحيوي الى علائق الحملان والماعز. وفي هذه الراسة بالرجم من عدم معنوية الفرق بين إضافة الفحم الحيوي وبدون الفحم الحيوي الا انه هناك تحسن بنسبة 18\% من خلال إضافة الفحم الحيوي.

كما اشارت النتائج في الجدول ذاته بعدم وجود فروقات بين مجموعتي الحملان المقدم لها الفحم الحيوي والحملان غير المقدم لها فحم حيوي في كل من معدل استهلاك العلف المركز (1309.61 , 1287.23) جم ومعدل استهلاك التبن(369.95, 387.66) جم على التوالي في حين كانت الاختلافات واضحة حسابيا لصالح مجموعة الحملان المغذات على فحم حيوي مقارنة مع مجموعة الحملان المغذات بدون فحم حيوي في كل من كفاءة التحويل الغذائي على اساس العلف الكلي ( 14.47 , 12.21 ) جم مادة جافة /جم زيادة وزنية.

عدم وجود فروقات معنوية اثر معاملة التبن باليوريا (جدول3) بين مجموعة الحملان المتتاولة التبن المعامل باليوريا ومجموعة الحملان

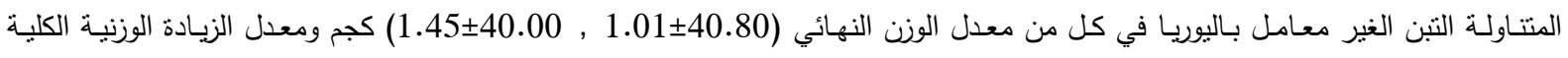

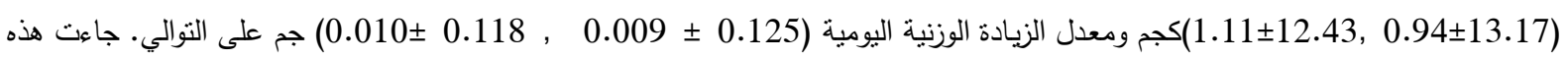
النتائج متفقه مع Hang واخرون (2018) اذ اثـاروا الى عدم وجود تأثير معنوي عند اضافه اليوريا الى عليقة الماعز في بعض الصفات الإنتاجية واثــار saroen و Preston (2018) بعدم وجود تأثثر معنوي لبلوكات البوريا و المولاس عنده اضــافتها الى عليقه الابقار في بعض الصــفات الإنتاجية. واختلفت مع فايز وسـليم (2017) حيث اثــاروا عند تغذية حملان البربري على التبن المعامل باليوريا الي فروقات معنويه في معدل 
اسـتهلاك العلف ومعدل الزيادة الوزنية اليومية وكفاءه التحويل الغذائي بين المعاملات. كما وجد جمعه وحميد (2017) حيث وجدوا فروقات معنويه في بعض الصفات الإنتاجية عند تغذيه الاغنام العواسي على التبن المعامل باليوريا. كما أظهرت النتائج أيضا في الجدول ذاته وجود فروقات معنوية واضحة في معدل استهلالك العلف المركز لصالح مجموعة الحملان التي أعطيت

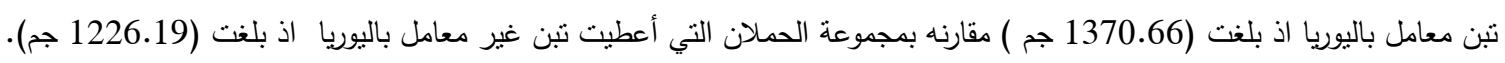

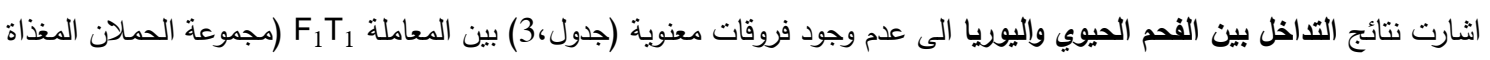

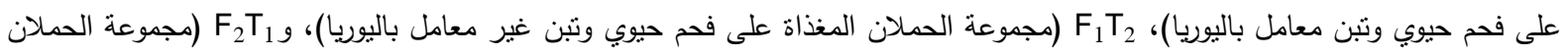

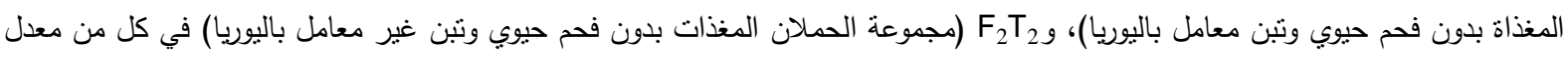

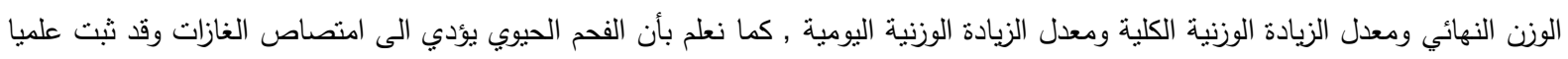

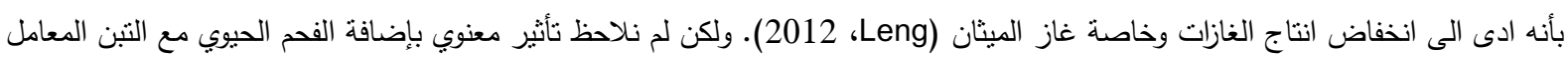

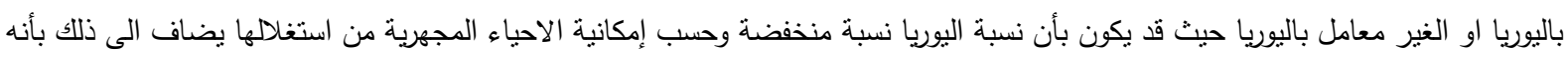

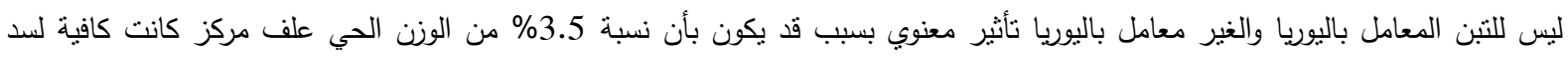
احتياجات الحيوان من المواد الغذائية.

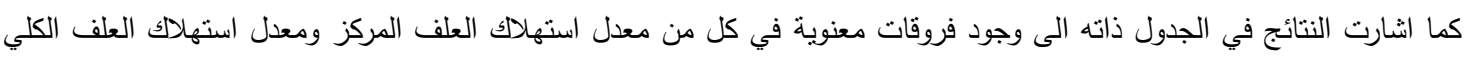

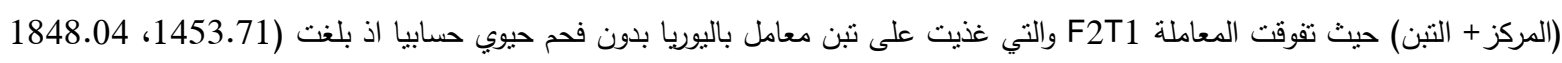

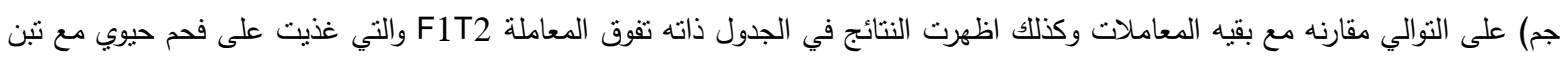

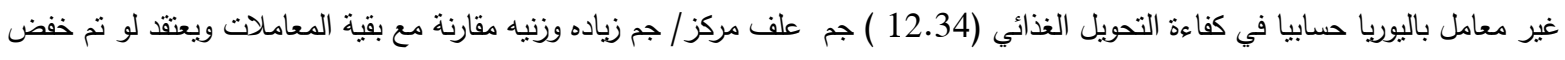

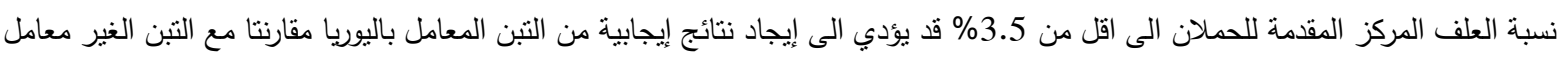
باليوريا بالأخص عند إضافة الفحم الحيوي الى التبن المعامل باليوريا.

نتائج صفات الذبيحة:

اظهرت نتائج التحليل الاحصائي تأثير الفحم الحيوي في الجدول رقم (4) عدم وجود فروقات معنويه بين مجموعتي الحملان المقدم لها عليقة

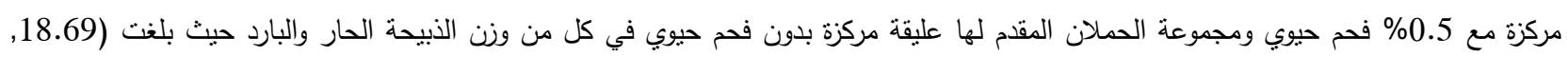

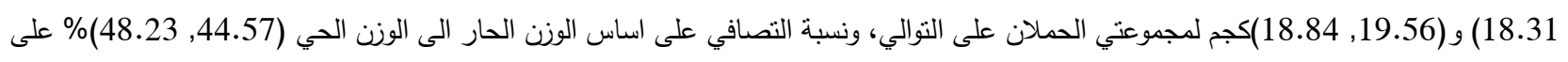

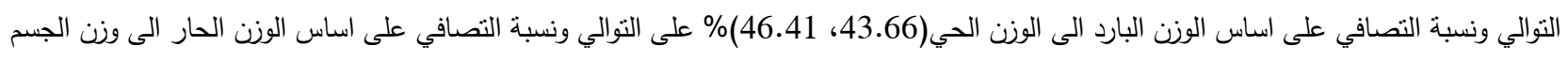

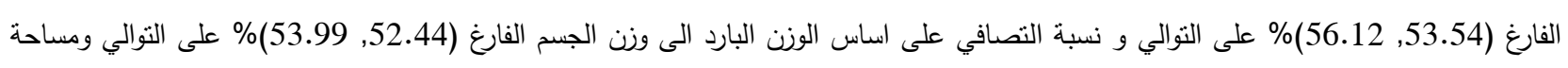

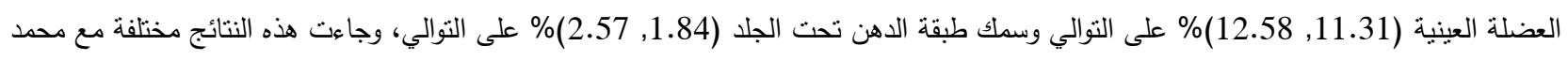
امين وشجاع (2019) حيث أثاروا الى وجود فروقات معنويه في بعض صفات الذيبحة عند تغذيه الحملان العواسي على مستويات مختلفة من الفحم

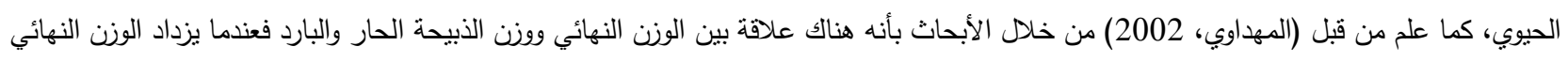

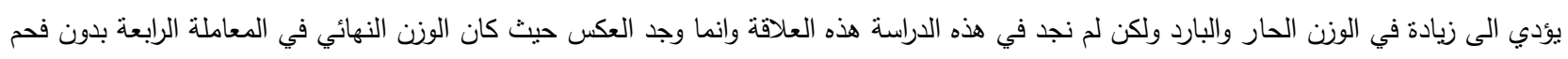

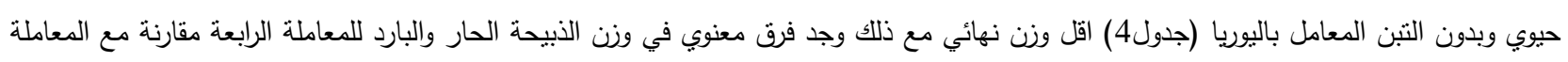
الثانية (إضافة فحم حيوي مع التنبن الغير معامل باليوريا).

عدم وجود فروقات معنوية أثير استعمال اليوريا في معاملة التبن (جدول4) بين مجموعتي الحملان التي أعطيت تبن معامل باليوريا(T)

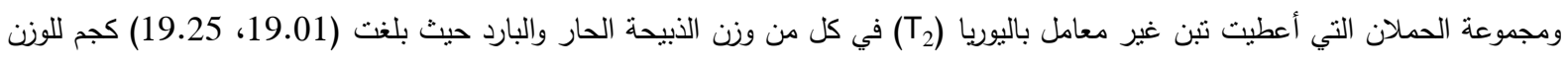

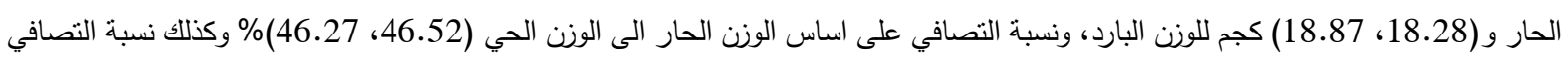

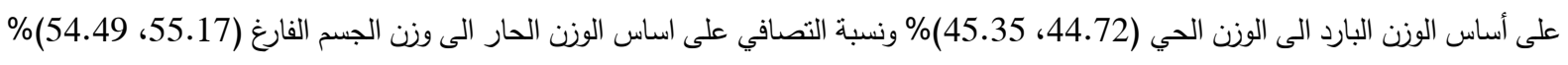


ونسبة التصافي على أساس الوزن البارد الى الوزن الجسم الفارغ (53.05، 53.39)\% على التوالي وكنلك مساحة العضلة العينية (12.84، 11.06) وسمك طبقه الدهن تحت الجلد (2.01، 2.95)\% على التوالي.

تأثير التذاخل بين الفحم الحيوي واليوريا (جدول، 4) , حيث اظهرت نتائج التحليل الاحصائي تفوق المعاملة الرابعة (F الحملان المغذاة بدون فحم حيوي وتبن غير معامل باليوريا) معنويا عند مستوى (20.05>p) في كل من الوزن الحار (20.41)كجم مقارنة مع المعاملة الثانية F F F Fمعة الحملان المغذاة على فحم حيوي وتبن غير معامل باليوريا) حيث بلغت (18.10) كجم وكذلك تفوقت المعاملة الرابعة (F $\left.F_{2} T_{2}\right)$

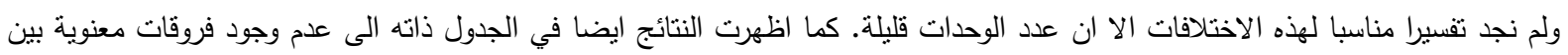
المعاملات الاربعة في كل من نسبة التصافي على اساس الوزن الحار الى الوزن الحي و سبة التصافي على اساس الوزن البارد الى الوزن الحي

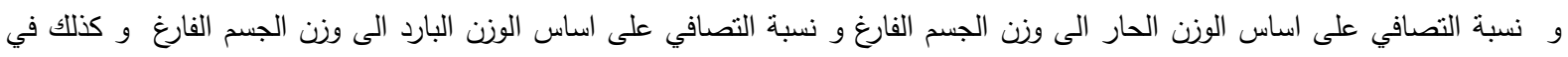
مساحة العضلة العينية ، في حين بينت النتائج تفوق المعاملة (F)

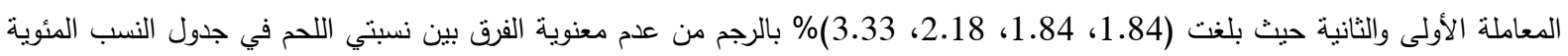
لأنسجة الفذذ (محمد امين و شجاع , 2019) الا أنه هناك ارتفاع حسابي في نسبة اللحم للمعاملة الثانية مقارنة مع المعاملة الرابعة يقابلها انخفاض في نسبة الدهن للمعاملة الثانية مقارنة مع المعاملة الرابعة وهذا قد يفسر ارتفاع سمك طبقة الدهن في المعاملة الرابعة (Fل

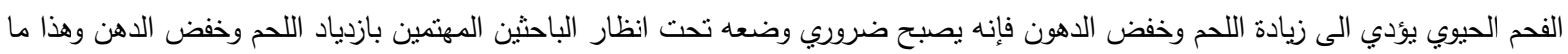
أكده محمد امين وشجاع (2019) بارتفاع نسبة اللحم الى الثحم عند استخدام 0.50 فحم حيوي. 
جدول 3. تأثير مستويين من الفحم الحيوي تحت مستويين من التنن المعامل باليوريا والثبن غير المعامل باليوريا على بعض الصفات الإنثاجية للحملان العواسي

\begin{tabular}{|c|c|c|c|c|c|c|c|c|c|}
\hline كم عفاءة التحويل & 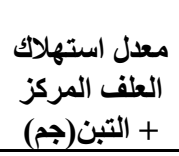 & معدل استهلاك & 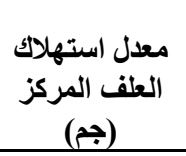 & $\begin{array}{c}\text { الوزنية/اليومية } \\
\text { (جيادة } \\
\text { (جيادة }\end{array}$ & معدل الزيادة الوزنية & النهائي(كج) الوز) & الابتدائي(كجم) & المشاهدات & العوأثرة \\
\hline 12.21 & 1674.90 & 387.66 & 1287.23 & $0.008 \pm 0.137$ & $13.44 \pm 0.89$ & $41.05 \pm 1.07$ & $27.61 \pm 0.56$ & 8 & F1 \\
\hline 14.47 & 1679.57 & 369.95 & 1309.61 & $0.116 \pm 0.011$ & $12.17 \pm .111$ & $1.38 \pm 39.75$ & $27.57 \pm .050$ & 8 & F2 \\
\hline 1 & 1 & 1 & 1 & م.غ & م.غ & م.غ & مغ & \multicolumn{2}{|c|}{ مستوى المعنوية } \\
\hline 14.12 & 1765.66 & 395 & 1370.66 & $0.009 \pm 0.125$ & $13.17 \pm 0.94$ & $1.014 \pm 0.80$ & $27.62 \pm 0.54$ & 8 & T1 \\
\hline 13.46 & 1588.80 & 362.61 & 1226.19 & $0.118 \pm 0.010$ & $12.43 \pm 1.11$ & $40.00 \pm 1.45$ & $27.56 \pm 0.54$ & 8 & $\mathbf{T} 2$ \\
\hline I & I & I & I & م.غ & ع.غ & م.غ & م.غ & \multicolumn{2}{|c|}{ مستوى المعنوية } \\
\hline 14.02 & 1683.19 & 395.66 & 1287.52 & $0.120 \pm 0.015$ & $12.65 \pm 1.62$ & $40.30 \pm 1.68$ & $27.65 \pm 0.92$ & 4 & F1T1 \\
\hline 12.34 & 1666.61 & 379.66 & 1286.95 & $0.007 \pm 0.135$ & $14.22 \pm 0.81$ & $41.80 \pm 1.47$ & $27.57 \pm 0.86$ & 4 & F1T2 \\
\hline 14.21 & 1848.04 & 394.33 & 1453.71 & $0.130 \pm 0.011$ & $13.70 \pm 1.14$ & $41.30 \pm 1.32$ & $27.60 \pm 0.74$ & 4 & F2T1 \\
\hline 14.96 & 1511 & 345.57 & 1165.42 & $0.016 \pm 0.101$ & $10.65 \pm 1.72$ & $38.20 \pm 2.35$ & $27.55 \pm 0.78$ & 4 & F2T2 \\
\hline I & I & I & I & غ غ. & غ غ. م & غ & غ غ & \multicolumn{2}{|c|}{ مستوى المعنوية } \\
\hline
\end{tabular}

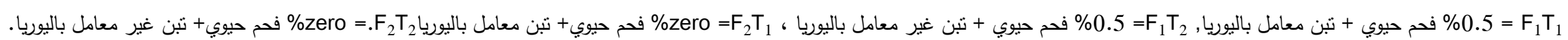


جدول (4) تأثثر استعمال عليقة مركزة مع الفحم الحيوي والتبن المعامل باليوريا وغير المعامل على بعض صفات الذبيحة للأغنام العواسي

\begin{tabular}{|c|c|c|c|c|c|c|c|c|c|}
\hline سمك الدهن تحت & مساحة العضلة & 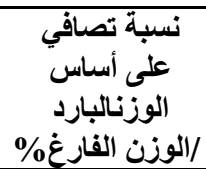 & 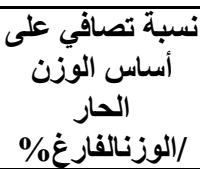 & 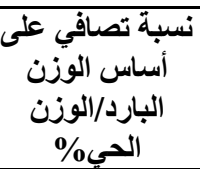 & نلى أسبة التصاس الوزّي & وزن الأبيحة & وزن الأبيحة (كجم) & المشاهدات & العوامل المؤثرة \\
\hline $2.30 \pm 0.26$ & $11.95 \pm 1.03$ & $53.22 \pm 0.81$ & $54.83 \pm 0.72$ & $45.04 \pm 0.95$ & $46.40 \pm 0.78$ & $18.57 \pm 0.65$ & $19.13 \pm 0.51$ & 8 & المتّأ القياسئ العام \\
\hline $1.84 \pm 0.17$ & $11.31 \pm 1.47$ & $52.44 \pm 0.58$ & $53.54 \pm 0.58$ & $43.66 \pm 0.73$ & $44.57 \pm 0.70$ & $18.31 \pm 0.49$ & $18.69 \pm 0.47$ & 4 & $\mathbf{F}_{1}$ \\
\hline $2.75 \pm 0.39$ & $12.58 \pm 0.50$ & $53.99 \pm 1.36$ & $56.12 \pm 0.79$ & $46.41 \pm 1.37$ & $48.23 \pm 0.93$ & $18.84 \pm 0.74$ & $19.56 \pm 0.52$ & 4 & $\mathbf{F}_{2}$ \\
\hline غ. & غ. & غ. & غ. & غ.غ & غ. & غ.غ & غ.غ & \multicolumn{2}{|c|}{ مستوى المعنوية } \\
\hline $2.01 \pm 0.27$ & $12.84 \pm 1.13$ & $53.05 \pm 1.04$ & $55.17 \pm 0.71$ & $44.72 \pm 0.99$ & $46.52 \pm 0.95$ & $18.28 \pm 0.53$ & $19.01 \pm 0.33$ & 4 & $\overline{T_{1}}$ \\
\hline $2.59 \pm 0.44$ & $11.06 \pm 0.94$ & $53.39 \pm 1.22$ & $54.49 \pm 1.22$ & $45.35 \pm 1.62$ & $46.27 \pm 1.63$ & $18.87 \pm 0.71$ & $19.25 \pm 0.71$ & 4 & $\mathbf{T}_{2}$ \\
\hline م.غे & غ.غ & م.غ & غ غ & غ.غ & م.غ & م.غ & م.غ & \multicolumn{2}{|c|}{ مستوى المعنوية } \\
\hline $\begin{array}{c}1.84 \pm 0.41 \\
B\end{array}$ & $2.76 \pm 12.76$ & $53.33 \pm 0.13$ & $54.38 \pm 0.32$ & $44.34 \pm 1.00$ & $45.21 \pm 0.87$ & $\begin{array}{c}18.92 \pm 0.76 \\
\mathrm{Ab}\end{array}$ & $\begin{array}{c}19.29 \pm 0.71 \\
\mathrm{Ab}\end{array}$ & 2 & $\mathbf{F}_{1} \mathbf{T}_{1}$ \\
\hline $\begin{array}{c}1.84 \pm 0.12 \\
\text { B }\end{array}$ & $9.86 \pm 1.10$ & $51.56 \pm 0.70$ & $52.70 \pm 0.72$ & $42.98 \pm 1.15$ & $43.93 \pm 1.18$ & $\begin{array}{c}17.71 \pm 0.39 \\
\text { B }\end{array}$ & $\begin{array}{c}18.10 \pm 0.40 \\
\mathrm{~B}\end{array}$ & 2 & $\mathbf{F}_{1} \mathbf{T}_{2}$ \\
\hline $\begin{array}{c}2.180 .47 \pm \\
\mathrm{Ab}\end{array}$ & $12.91 \pm 0.16$ & $52.76 \pm 2.53$ & $55.95 \pm 1.31$ & $45.11 \pm 2.15$ & $47.84 \pm 1.11$ & $\begin{array}{c}17.65 \pm 0.55 \\
\text { B }\end{array}$ & $\mathrm{ab} 18.10 \pm 0.12$ & 2 & $\mathbf{F}_{2} \mathbf{T}_{1}$ \\
\hline $\begin{array}{c}3.33 \pm 0.26 \\
\mathrm{~A}\end{array}$ & $12.25 \pm 1.12$ & $55.23 \pm 1.30$ & $56.28 \pm 1.41$ & $47.71 \pm 1.81$ & $48.62 \pm 1.93$ & $\begin{array}{c}20.03 \pm 0.43 \\
\mathrm{~A}\end{array}$ & $\begin{array}{c}20.41 \pm 0.47 \\
\mathrm{~A}\end{array}$ & 2 & $\mathbf{F}_{2} \mathbf{T}_{2}$ \\
\hline$*$ & م.غ & (غ & غ.غ & غ.غ & غ.غ & $*$ & $*$ & \multicolumn{2}{|c|}{ مستوى المعنوية } \\
\hline
\end{tabular}

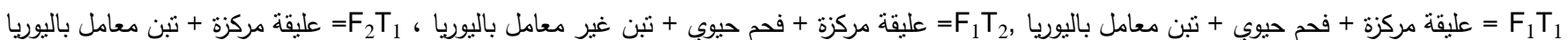

عليقة مركزة +تبن غبر معامل باليوريا $=F_{2} T_{2}$ 


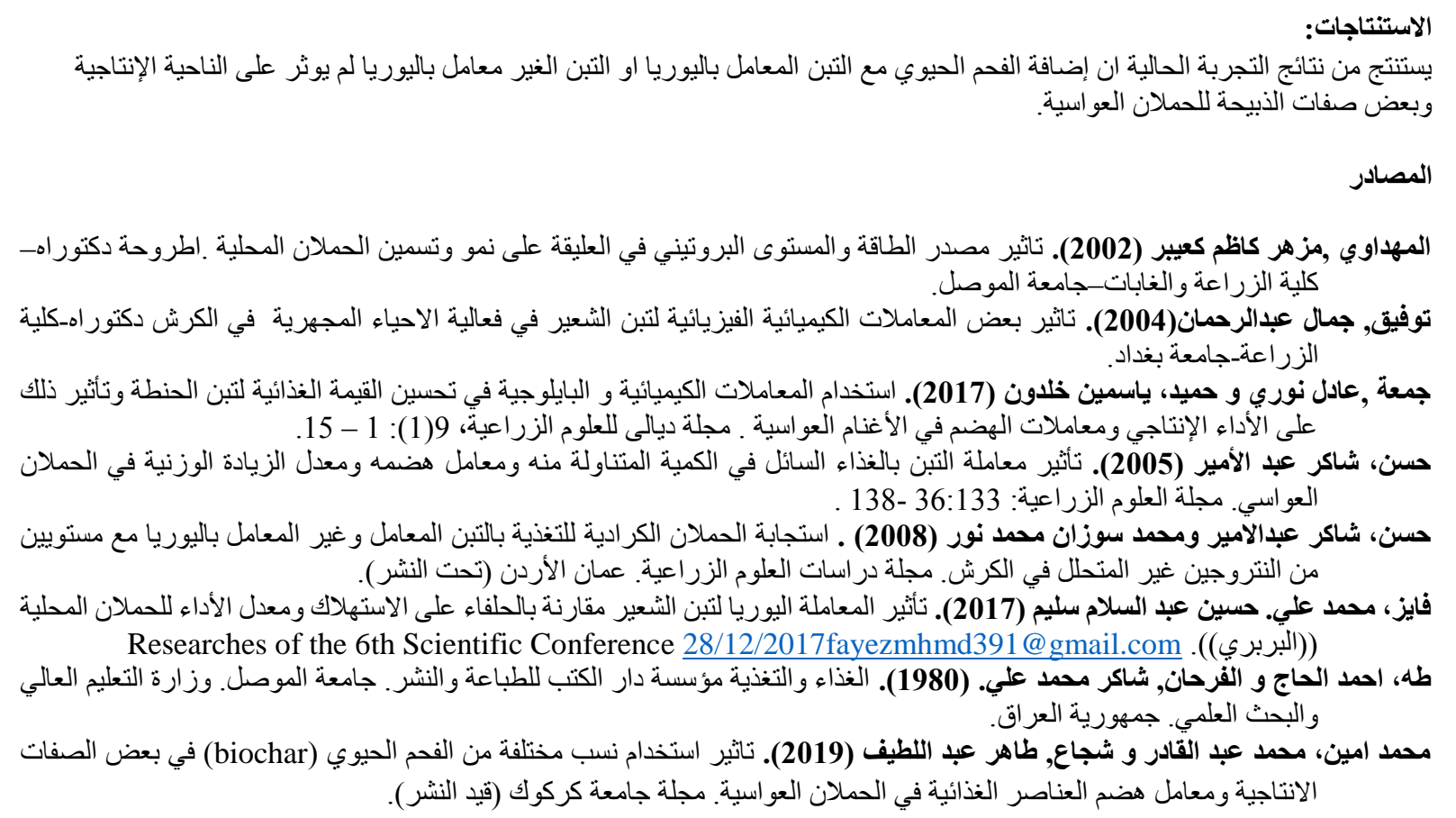

A.O.A.C. (1990). official methods of analysis 15 th end. Association of official analytical chemists , Arlington, Virginia.

Duncan, D. (1955). Multiple range and multiple F-test.Biometrics.11:1-24

Field ,R . A. ;Kamp ,J.D. ;and Varney, W.Y.(1963).Carcass evaluation of lambs from selected sires .J. Animal Sci., 2:364-367.

Hang Thuy, L.T.; Preston1, T. R. ; Nguyen, X. B. and Dung, D. V.(2019) Effect of biochar on growth and methane emissions of goats fed fresh cassava foliage.Livestock Research for Rural Development 31 (5

Hang Thuy, L. T.; Preston, T. R.; Leng, R. A. and Ba, N. X. (2018). Effect of biochar and water spinach on feed intake, digestibility and $\mathrm{N}$-retention in goats fed urea-treated cassava stems. Livestock Research for Rural Development, 30 .

Leng, R. A., Preston, T. R., \&Inthapanya, S. (2012).Biochar reduces enteric methane and improves growth and feed conversion in local "Yellow" cattle fed cassava root chips and fresh cassava foliage. Livestock Research for Rural Development, 24(11).

MAFF, (1984) Ministry of Agric. Fisheris and food. Energy allowance And feeding system for ruminants ( $2^{\text {nd }}$ end) don .HerMaestys stat. office

Mohammed Ameen, M. A .and Shujaa,T.A.(2019b) Effect of adding biochar to ration on productive performance and some carcass characteristics of Iraqi Awassi lambs .Tikrit Journal for Agricultural Sci.19:4

Riley, M. L. ; Field, R. A. and Neims A.H. .(1966) Comparison of two methods of measuring the area of longissimus dorsi muscle. J. Anim. Sci. $25: 587-592$.

Saroen, P. and Preston, T. R. (2018) Rice distillers' byproduct and molasses-urea blocks containing biochar improved the growth performance of local Yellow cattle fed ensiled cassava roots, cassava foliage and rice straw .Livestock Research for Rural Development. Volume 27, Article \#58.Retrieved March 20, 2015, from http://www.lrrd.org//rrd27/3/sili27058.html.

SAS,( 2012) Statistical Analysis System, User's Guide. Statistical. Version $9.1^{\text {th }}$ ed. SAS. Inst. Inc. Cary. N.C. USA.

Silivong, P. and Preston, T. R. (2015) Growth performance of goats was improved when a basal diet of foliage of Bauhinia acuminata was supplemented with water spinach and biochar.Livestock Research for Rural Development.Volume 27, Article \#58.Retrieved March 20, 2015, from http://www.lrrd.org/lrrd27/3/sili27058.html 


\section{Effect of Adding Biochar to Treated or Untreated Straw with Urea on Production Performance and Some Carcass Characteristics of Awassi Lambs \\ Afraah M. Mohammed Al-Sammari \\ afrah_mustafa@tu.edu.iq \\ Taher A. Shujaa \\ tshujaa@yahoo.com \\ Basam S. ALRazaq \\ Tikrit University - Collage of Agriculture - Animals Production Dept.}

16 Awassi lambs were used in this experiment, aged 7-8 months and average weight $27.55 \pm 0.78 \mathrm{Kg}$. Lambs were divided in to four groups according to their weight, each group had four lambs, then distributed randomly on groups. Lambs fed diet with $3.5 \%$ concentrate of body weight, treatments as below.

First treatment $=$ concentrate $+0.5 \%$ biochar + treated straw with urea

Second treatment $=$ concentrate $+0.5 \%$ biochar + untreated straw with urea,

Third treatment $=$ concentrate + zero $\%$ biochar + urea treated straw,

Fourth treatment $=$ concentrate + zero $\%$ biochar + untreated urea straw

The results analyzed according to random design $(2 \times 2)$. Results showed no significant difference in productivn performance (average final weight, total body gain, average daily gain, feed intake and feed conversion), also there were no significant difference in carcass characteristics of Awassi lambs(percentage dressing and Rip-eye muscle area) although there were significant improvement in the fourth treatment in hot , cold and fat thickness under skin.

Keywords: biochar, straw treated urea, Awassi lambs, carcass Characteristics. 